\title{
Positive solutions for a class of fractional boundary value problems with fractional boundary conditions
}

\author{
I. Azman ${ }^{a}$, M. Jlelia , B. López ${ }^{\text {b }}$ K. Sadarangani ${ }^{b}$, B. Samet ${ }^{a, *}$ \\ ${ }^{a}$ Department of Mathematics, College of Science, King Saud University, P. O. Box 2455, Riyadh, 11451, Saudi Arabia. \\ ${ }^{b}$ Departamento de Matematicas, Universidad de Las Palmas de Gran Canaria, Campus de Tafira Baja, 35017 Las Palmas de Gran \\ Canaria, Spain.
}

Communicated by D. Baleanu

\begin{abstract}
In this paper, we study the solvability of a nonlinear fractional differential equation under fractional integral boundary conditions. Via a mixed monotone operator method, some new results on the existence and uniqueness of a positive solution for the considered model are obtained. Moreover, we provide iterative sequences for approximating the solution. Some examples are also presented in order to illustrate the obtained result.
\end{abstract}

Keywords: Fractional boundary value problem, fractional integral boundary condition, mixed monotone operator. 2010 MSC: 34A08, 31B10, 47H07.

(C)2018 All rights reserved.

\section{Introduction}

Fractional differential equations have received increasing attention during recent years due to their wide range of applications in a variety of disciplines in science and engineering, such as physics, control theory, chemistry, biology, ecology, aerodynamics, etc. It was shown that derivatives and integrals of fractional type provide an adequate mathematical modeling of real objects and processes. For details, we refer the reader to $[6,12,13,15,19,21-23]$ and references therein.

Recently, basing on nonlinear analysis tools, several existence results for different classes of fractional boundary value problems were established. In this direction, we refer to $[1-5,7,8,10,17,18,20,22,26-$ 30] and references therein. In particular, many works have been devoted to the study of the existence of solutions for fractional differential equations under different kinds of integral (or fractional integral) boundary conditions, see $[2-5,7,8,17,26-29]$ and references therein. The motivation of a such study

\footnotetext{
*Corresponding author

Email addresses: ibtehalazman@yahoo.com (I. Azman), jleli@ksu.edu.sa (M. Jleli), blopez@dma.ulpgc.es (B. López), ksadaran@dma.ulpgc.es (K. Sadarangani), bsamet@ksu.edu.sa (B. Samet)

doi: $10.22436 /$ jnsa.011.02.06
}

Received: 2017-10-05 Revised: 2017-12-17 Accepted: 2017-12-21 
is due to the importance and numerous applications of integral boundary conditions in different fields, such as blood flow problems, chemical engineering, thermo-elasticity, etc. See, for example [2, 11, 24, 25].

In this paper, we are concerned with the solvability of a class of nonlinear fractional differential equations with fractional integral boundary conditions. More precisely, we are concerned with the fractional boundary value problem

$$
\left\{\begin{array}{l}
D_{0}^{\alpha} u(t)+f(t, u(t),(H u)(t))+g(t, u(t))=0, \quad 0<t<1, \\
u(0)=u^{\prime}(0)=0, u(1)=\lambda\left(\left(I_{0}^{\beta} u\right)(1)+\left(I_{0}^{\gamma} u\right)(1)\right),
\end{array}\right.
$$

where $2<\alpha<3, \lambda>0, \beta, \gamma \geqslant 0, D_{0}^{\alpha}$ is the Riemann-Liouville left-sided fractional derivative of order $\alpha$, $\mathrm{I}_{0}^{\mathrm{q}}, \mathrm{q} \in\{\beta, \gamma\}$ is the Riemann-Liouville left-sided fractional integral of order $\mathrm{q}, \mathrm{f}:[0,1] \times[0, \infty) \times[0, \infty) \rightarrow$ $[0, \infty), \mathrm{g}:[0,1] \times[0, \infty) \rightarrow[0, \infty)$, and $\mathrm{H}: \mathrm{C}([0,1] ; \mathbb{R}) \rightarrow \mathrm{C}([0,1] ; \mathbb{R})$ is a certain operator (not necessarily linear). We provide sufficient conditions for the existence and uniqueness of positive solutions for Problem (1.1). Moreover, we present an iterative algorithm that converges to the solution. Some examples are also presented in order to illustrate the obtained result. Our approach is based on a mixed monotone operator method introduced in [29].

The paper is organized as follows. In Section 2, we recall briefly some basic concepts on fractional calculus. In Section 3, we define the adequate functional space on which problem (1.1) is posed, we compute its Green's function, and then we obtain an integral formulation of the problem. In Section 4, we recall the basic tools related to the mixed monotone operator method. In Section 5, we state and prove our main result (Theorem 5.1). In Section 6, several examples are presented in order to illustrate the obtained result. Finally, in Section 7, a comparison with existence results from the literature is presented.

\section{Preliminaries on fractional calculus}

Let $(a, b) \in \mathbb{R}^{2}$ with $a<b$.

Definition 2.1 ([19]). Let $f \in \mathrm{L}^{1}([a, b] ; \mathbb{R})$. The Riemann-Liouville left-sided fractional integral of order $\alpha>0$ of $f$ is defined by

$$
\left(I_{a}^{\alpha} f\right)(t)=\frac{1}{\Gamma(\alpha)} \int_{a}^{t} \frac{f(s)}{(t-s)^{1-\alpha}} d s, \quad \text { a.e. } t \in[a, b],
$$

where $\Gamma$ is the Gamma function. For $\alpha=0$, we take $I_{a}^{0} f=f$.

Lemma 2.2 ([19]). If $\alpha_{1}, \alpha_{2}>0$, then

$$
\left(I_{a}^{\alpha_{1}} I_{a}^{\alpha_{2}} f\right)(t)=\left(I_{a}^{\alpha_{1}+\alpha_{2}} f\right)(t), \quad \text { a.e. } t \in[a, b],
$$

for every $f \in \mathrm{L}^{1}([\mathrm{a}, \mathrm{b}] ; \mathbb{R})$.

Lemma 2.3 ([16]). If $\alpha>0, p \geqslant 1$, and $p>\frac{1}{\alpha}$, then $\mathrm{I}_{a}^{\alpha} \mathrm{f} \in \mathrm{C}([\mathrm{a}, \mathrm{b}] ; \mathbb{R})$ for every $f \in \mathrm{L}^{\mathrm{p}}([\mathrm{a}, \mathrm{b}] ; \mathbb{R})$.

Lemma 2.4 ([19]). Let $\mu, \alpha>0$. Then

$$
\mathrm{I}_{\mathrm{a}}^{\mu}(\mathrm{t}-\mathrm{a})^{\alpha-1}=\frac{\Gamma(\alpha)}{\Gamma(\alpha+\mu)}(\mathrm{t}-\mathrm{a})^{\alpha+\mu-1}, \quad \mathrm{t}>\mathrm{a} .
$$

Let $n \in \mathbb{N}, n \geqslant 1$. By $A C^{n}([a, b], \mathbb{R})$, we denote the space of all functions $f:[a, b] \rightarrow \mathbb{R}$ that are absolutely continuous together with the classical derivatives $f, D f, \ldots, D^{n-1} f$.

Definition 2.5 ([16]). Let $n-1<\alpha<n$ for some $n \in \mathbb{N}, n \geqslant 1$, and $f \in L^{1}([a, b] ; \mathbb{R})$. We say that $f$ possesses the left-sided Riemann-Liouville derivative $D_{a}^{\alpha}$ of order $\alpha$ on the interval $[a, b]$ if $I_{a}^{n-\alpha_{f}} \in$ $A C^{n}([a, b], \mathbb{R})$. By this derivative we mean the derivative $D^{n}\left(I_{a}^{n-\alpha} f\right)$, i.e.,

$$
D_{a}^{\alpha} f(t)=\frac{1}{\Gamma(n-\alpha)} D^{n}\left(\int_{a}^{t} \frac{f(s)}{(t-s)^{1-n+\alpha}} d s\right), \quad \text { a.e. } t \in[a, b] .
$$


Lemma 2.6 ([16]). Let $\mathrm{n}-1<\alpha<\mathrm{n}$ for some $\mathrm{n} \in \mathbb{N}, \mathrm{n} \geqslant 2$, and $\mathrm{f} \in \mathrm{L}^{1}([\mathrm{a}, \mathrm{b}] ; \mathbb{R})$. Then $\mathrm{f}$ has the left-sided Riemann-Liouville derivative $\mathrm{D}_{\mathrm{a}}^{\alpha} \mathrm{f}$ of order $\alpha$ on the interval $[\mathrm{a}, \mathrm{b}]$ if and only if there exist constants $\mathrm{c}_{0}, \mathrm{c}_{1}, \ldots, \mathrm{c}_{\mathrm{n}-1} \in \mathbb{R}$ and a function $\varphi \in \mathrm{L}^{1}([\mathrm{a}, \mathrm{b}] ; \mathbb{R})$ such that

$$
\begin{aligned}
f(t)= & \frac{c_{0}}{\Gamma(\alpha-n+1)}(t-a)^{\alpha-n}+\frac{c_{1}}{\Gamma(\alpha-n+2)}(t-a)^{\alpha-n+1} \\
& +\cdots+\frac{c_{n-1}}{\Gamma(\alpha)}(t-a)^{\alpha-1}+I_{a}^{\alpha} \varphi(t), \quad \text { a.e. } t \in[a, b] .
\end{aligned}
$$

In such a case, we have

$$
c_{i}=D^{i}\left(I_{a}^{n-\alpha} f\right)(a), \quad i=0,1,2, \ldots, n-1
$$

and

$$
D_{a}^{\alpha} f(t)=\varphi(t), \quad \text { a.e. } t \in[a, b]
$$

\section{Integral representation of solutions}

In order to obtain an integral formulation of problem (1.1), we need the computation of its Green's function. Here, we compute rigorously the Green's function associated to problem (1.1) with the adequate functional spaces.

By $A C_{a}^{\alpha}([a, b] ; \mathbb{R})$, we denote the set of functions $f:[a, b] \rightarrow \mathbb{R}$ that belong to $L^{1}([a, b] ; \mathbb{R})$, and having the representation (2.1). We introduce the functional space $X_{a}^{\alpha}([a, b] ; \mathbb{R})$ defined by

$$
X_{a}^{\alpha}([a, b] ; \mathbb{R})=A C_{a}^{\alpha}([a, b] ; \mathbb{R}) \cap C^{1}([a, b] ; \mathbb{R})
$$

We have the following characterization of the above functional space in the case $2<\alpha<3$.

Lemma 3.1. Let $2<\alpha<3$. For every $u \in X_{0}^{\alpha}([0,1] ; \mathbb{R})$, we have

$$
u(0)=u^{\prime}(0)=0 .
$$

Proof. Let $u \in X_{0}^{\alpha}([0,1] ; \mathbb{R})$. Then the function $u$ has the representation (2.1) with $n=3$, that is,

$$
\mathrm{u}(\mathrm{t})=\frac{\mathrm{c}_{0}}{\Gamma(\alpha-2)} \mathrm{t}^{\alpha-3}+\frac{\mathrm{c}_{1}}{\Gamma(\alpha-1)} \mathrm{t}^{\alpha-2}+\frac{\mathrm{c}_{2}}{\Gamma(\alpha)} \mathrm{t}^{\alpha-1}+\frac{1}{\Gamma(\alpha)} \int_{0}^{\mathrm{t}} \varphi(\mathrm{s})(\mathrm{t}-\mathrm{s})^{\alpha-1} \text { ds, } \quad \text { a.e. } \mathrm{t} \in[0,1],
$$

where $c_{i} \in \mathbb{R}, i=0,1,2$, and $\varphi=D_{0}^{\alpha} u \in L^{1}([0,1] ; \mathbb{R})$. Since $u$ is continuous at 0 , we have

$$
\lim _{t \rightarrow 0^{+}} u(t)=u(0) \text {. }
$$

On the other hand, since $2<\alpha<3$, we have

$$
\lim _{t \rightarrow 0^{+}} u(t)=\lim _{t \rightarrow 0^{+}}\left(\frac{c_{0}}{\Gamma(\alpha-2)} t^{\alpha-3}+\frac{1}{\Gamma(\alpha)} \int_{0}^{t} \varphi(s)(t-s)^{\alpha-1} d s\right) .
$$

Observe that

$$
\left|\int_{0}^{t} \varphi(s)(t-s)^{\alpha-1} d s\right| \leqslant \int_{0}^{t}|\varphi(s)| d s
$$

Therefore,

$$
\lim _{t \rightarrow 0^{+}} \int_{0}^{t} \varphi(s)(t-s)^{\alpha-1} d s=0
$$

Hence, we have

$$
u(0)=\lim _{t \rightarrow 0^{+}} u(t)=\lim _{t \rightarrow 0^{+}} \frac{c_{0}}{\Gamma(\alpha-2)} t^{\alpha-3},
$$


which yields $c_{0}=0$ and $u(0)=0$. Next, it follows that

$$
u(t)=\frac{c_{1}}{\Gamma(\alpha-1)} t^{\alpha-2}+\frac{c_{2}}{\Gamma(\alpha)} t^{\alpha-1}+\frac{1}{\Gamma(\alpha)} \int_{0}^{t} \varphi(s)(t-s)^{\alpha-1} d s, \quad \text { a.e. } t \in[0,1] .
$$

Differentiating with respect to $t$, we get

$$
u^{\prime}(t)=\frac{c_{1}(\alpha-2)}{\Gamma(\alpha-1)} t^{\alpha-3}+\frac{c_{2}(\alpha-1)}{\Gamma(\alpha)} t^{\alpha-2}+\frac{(\alpha-1)}{\Gamma(\alpha)} \int_{0}^{t} \varphi(s)(t-s)^{\alpha-2} d s, \quad \text { a.e. } t \in[0,1] .
$$

Since $u^{\prime}$ is continuous at 0 , we have

$$
\lim _{t \rightarrow 0^{+}} u^{\prime}(t)=u^{\prime}(0)
$$

On the other hand, we have

$$
\lim _{t \rightarrow 0^{+}} u^{\prime}(t)=\lim _{t \rightarrow 0^{+}}\left(\frac{c_{1}(\alpha-2)}{\Gamma(\alpha-1)} t^{\alpha-3}+\frac{(\alpha-1)}{\Gamma(\alpha)} \int_{0}^{t} \varphi(s)(t-s)^{\alpha-2} d s\right) .
$$

Similarly, we have

$$
\lim _{t \rightarrow 0^{+}} \int_{0}^{t} \varphi(s)(t-s)^{\alpha-2} d s=0
$$

which yields

$$
u^{\prime}(0)=\lim _{t \rightarrow 0^{+}} \frac{c_{1}(\alpha-2)}{\Gamma(\alpha-1)} t^{\alpha-3} .
$$

Hence, we obtain $c_{1}=0$ and $\mathfrak{u}^{\prime}(0)=0$.

From Lemma 3.1, we deduce immediately the following characterization of the functional space $X_{0}^{\alpha}([0,1] ; \mathbb{R})$ for $2<\alpha<3$.

Lemma 3.2. Let $2<\alpha<3$. For every $u \in X_{0}^{\alpha}([0,1] ; \mathbb{R})$, there exists $c(u) \in \mathbb{R}$ such that

$$
\mathrm{u}(\mathrm{t})=\frac{\mathrm{c}(\mathrm{u})}{\Gamma(\alpha)} \mathrm{t}^{\alpha-1}+\left(\mathrm{I}_{0}^{\alpha} \varphi\right)(\mathrm{t}), \quad \text { a.e. } \mathrm{t} \in[0,1]
$$

where $\varphi=\mathrm{D}_{0}^{\alpha} \mathfrak{u} \in \mathrm{L}^{1}([\mathrm{a}, \mathrm{b}] ; \mathbb{R})$.

Lemma 3.3. Let $2<\alpha<3$ and $\beta, \gamma \geqslant 0$. For every $u \in X_{0}^{\alpha}([0,1] ; \mathbb{R})$, we have

$$
\left(I_{0}^{\beta} \mathrm{u}\right)(1)+\left(\mathrm{I}_{0}^{\gamma} \mathrm{u}\right)(1)=\left(\frac{1}{\Gamma(\alpha+\beta)}+\frac{1}{\Gamma(\alpha+\gamma)}\right) \mathrm{c}(\mathrm{u})+\int_{0}^{1}(1-\mathrm{s})^{\alpha-1}\left[\frac{(1-s)^{\beta}}{\Gamma(\alpha+\beta)}+\frac{(1-s)^{\gamma}}{\Gamma(\alpha+\gamma)}\right] \varphi(s) \mathrm{d},
$$

where $\varphi=\mathrm{D}_{0}^{\alpha} \mathrm{u} \in \mathrm{L}^{1}([\mathrm{a}, \mathrm{b}] ; \mathbb{R})$.

Proof. Let $u \in X_{0}^{\alpha}([0,1] ; \mathbb{R})$. Using Lemma 3.2, we obtain

$$
\left(I_{0}^{\beta} \mathrm{u}\right)(\mathrm{t})=\frac{\mathrm{c}(\mathrm{u})}{\Gamma(\alpha)} \mathrm{I}_{0}^{\beta} \mathrm{t}^{\alpha-1}+\left(\mathrm{I}_{0}^{\beta} \mathrm{I}_{0}^{\alpha} \varphi\right)(\mathrm{t}), \quad \text { a.e. } \mathrm{t} \in[0,1] .
$$

On the other hand, from Lemmas 2.4 and 2.2, we have

$$
\mathrm{I}_{0}^{\beta} \mathrm{t}^{\alpha-1}=\frac{\Gamma(\alpha)}{\Gamma(\alpha+\beta)} \mathrm{t}^{\alpha+\beta-1}
$$

and

$$
\left(\mathrm{I}_{0}^{\beta} \mathrm{I}_{0}^{\alpha} \varphi\right)(\mathrm{t})=\left(\mathrm{I}_{0}^{\alpha+\beta} \varphi\right)(\mathrm{t}), \quad \text { a.e. } \mathrm{t} \in[0,1] .
$$


Therefore,

$$
\left(I_{0}^{\beta} u\right)(t)=\frac{c(u)}{\Gamma(\alpha+\beta)} t^{\alpha+\beta-1}+\left(I_{0}^{\alpha+\beta} \varphi\right)(t), \quad \text { a.e. } t \in[0,1] .
$$

From Lemma 2.3 , we have $I_{0}^{\beta} u \in C([0,1] ; \mathbb{R})$. Hence,

$$
\left(I_{0}^{\beta} u\right)(1)=\lim _{t \rightarrow 1^{-}}\left(I_{0}^{\beta} u\right)(t)=\frac{c(u)}{\Gamma(\alpha+\beta)}+\frac{1}{\Gamma(\alpha+\beta)} \int_{0}^{1}(1-s)^{\alpha+\beta-1} \varphi(s) d s .
$$

Similarly, we obtain

$$
\left(\mathrm{I}_{0}^{\gamma} \mathrm{u}\right)(1)=\frac{\mathrm{c}(\mathrm{u})}{\Gamma(\alpha+\gamma)}+\frac{1}{\Gamma(\alpha+\gamma)} \int_{0}^{1}(1-\mathrm{s})^{\alpha+\gamma-1} \varphi(\mathrm{s}) \mathrm{d} s .
$$

Adding (3.1) to (3.2), the desired result follows.

Lemma 3.4. Let $2<\alpha<3, \lambda>0$, and $\beta, \gamma \geqslant 0$. Suppose that

$$
[C(\lambda, \alpha, \beta, \gamma)]^{-1}:=\frac{1}{\Gamma(\alpha)}-\frac{\lambda}{\Gamma(\alpha+\beta)}-\frac{\lambda}{\Gamma(\alpha+\gamma)} \neq 0 .
$$

Let $\mathrm{u} \in \mathrm{X}_{0}^{\alpha}([0,1] ; \mathbb{R})$ be such that

$$
u(1)=\lambda\left(\left(I_{0}^{\beta} \mathfrak{u}\right)(1)+\left(I_{0}^{\gamma} \mathfrak{u}\right)(1)\right)
$$

Then

$$
u(t)=\int_{0}^{1} G(t, s)[-\varphi(s)] d s, \quad t \in[0,1],
$$

where $\varphi=\mathrm{D}_{0}^{\alpha} \mathrm{u} \in \mathrm{L}^{1}([\mathrm{a}, \mathrm{b}] ; \mathbb{R})$ and

$$
G(t, s)=\frac{C(\lambda, \alpha, \beta, \gamma)}{\Gamma(\alpha)} \begin{cases}t^{\alpha-1}(1-s)^{\alpha-1} \psi(s)-\frac{1}{C(\lambda, \alpha, \beta, \gamma)}(t-s)^{\alpha-1}, & 0 \leqslant s \leqslant t \leqslant 1, \\ t^{\alpha-1}(1-s)^{\alpha-1} \psi(s), & 0 \leqslant t \leqslant s \leqslant 1,\end{cases}
$$

where

$$
\psi(s)=\left[\frac{1}{\Gamma(\alpha)}-\frac{\lambda(1-s)^{\beta}}{\Gamma(\alpha+\beta)}-\frac{\lambda(1-s)^{\gamma}}{\Gamma(\alpha+\gamma)}\right], \quad s \in[0,1] .
$$

Proof. From Lemma 3.2, we have

$$
u(t)=\frac{c(u)}{\Gamma(\alpha)} t^{\alpha-1}+\left(I_{0}^{\alpha} \varphi\right)(t), \quad \text { a.e. } t \in[0,1],
$$

where $\varphi=D_{0}^{\alpha} u \in L^{1}([a, b] ; \mathbb{R})$. On the other hand, since $u$ is continuous in $[0,1]$, the above equality can be extended to all the inteval $[0,1]$, i.e.,

$$
u(t)=\frac{c(u)}{\Gamma(\alpha)} t^{\alpha-1}+\left(I_{0}^{\alpha} \varphi\right)(t), \quad t \in[0,1]
$$

Taking $\mathrm{t}=1$, we obtain

$$
u(1)=\frac{c(u)}{\Gamma(\alpha)}+\frac{1}{\Gamma(\alpha)} \int_{0}^{1}(1-s)^{\alpha-1} \varphi(s) d s .
$$

Hence, by(3.4) and Lemma 3.3, we obtain

$$
\frac{c(u)}{\Gamma(\alpha)}+\frac{1}{\Gamma(\alpha)} \int_{0}^{1}(1-s)^{\alpha-1} \varphi(s) d s
$$




$$
=\left(\frac{\lambda}{\Gamma(\alpha+\beta)}+\frac{\lambda}{\Gamma(\alpha+\gamma)}\right) \mathrm{c}(\mathrm{u})+\int_{0}^{1}(1-\mathrm{s})^{\alpha-1}\left[\frac{\lambda(1-s)^{\beta}}{\Gamma(\alpha+\beta)}+\frac{\lambda(1-s)^{\gamma}}{\Gamma(\alpha+\gamma)}\right] \varphi(\mathrm{s}) \mathrm{ds},
$$

which from (3.3) yields

$$
c(u)=C(\lambda, \alpha, \beta, \gamma) \int_{0}^{1}(1-s)^{\alpha-1} \psi(s)[-\varphi(s)] d s
$$

Therefore, we obtain

$$
u(t)=\frac{C(\lambda, \alpha, \beta, \gamma)}{\Gamma(\alpha)} \int_{0}^{1} t^{\alpha-1}(1-s)^{\alpha-1} \psi(s)[-\varphi(s)] d s-\frac{1}{\Gamma(\alpha)} \int_{0}^{t}(t-s)^{\alpha-1}[-\varphi(s)] d s
$$

for every $t \in[0,1]$. Next, we have

$$
\begin{aligned}
u(t)= & \int_{0}^{t} \frac{C(\lambda, \alpha, \beta, \gamma)}{\Gamma(\alpha)}\left[t^{\alpha-1}(1-s)^{\alpha-1} \psi(s)-\frac{1}{C(\lambda, \alpha, \beta, \gamma)}(t-s)^{\alpha-1}\right][-\varphi(s)] d s \\
& +\int_{t}^{1} \frac{C(\lambda, \alpha, \beta, \gamma)}{\Gamma(\alpha)} t^{\alpha-1}(1-s)^{\alpha-1} \psi(s)[-\varphi(s)] d s, \quad t \in[0,1],
\end{aligned}
$$

which yields the desired result.

Lemma 3.5. Let $2<\alpha<3$ and $\beta, \gamma \geqslant 0$. Suppose that

$$
0<\lambda\left(\frac{1}{\Gamma(\alpha+\beta)}+\frac{1}{\Gamma(\alpha+\gamma)}\right)<\frac{1}{\Gamma(\alpha)} .
$$

Then

(i) $\mathrm{G}$ is continuous in $[0,1] \times[0,1]$;

(ii) for every $(t, s) \in[0,1] \times[0,1]$, we have

$$
t^{\alpha-1} \mu(s) \leqslant G(t, s) \leqslant t^{\alpha-1} v(s),
$$

where

$$
\mu(s)=\frac{C(\lambda, \alpha, \beta, \gamma)}{\Gamma(\alpha)}(1-s)^{\alpha-1}\left[\frac{\lambda}{\Gamma(\alpha+\beta)}\left(1-(1-s)^{\beta}\right)+\frac{\lambda}{\Gamma(\alpha+\gamma)}\left(1-(1-s)^{\gamma}\right)\right]
$$

and

$$
v(s)=\frac{C(\lambda, \alpha, \beta, \gamma)}{\Gamma(\alpha)}(1-s)^{\alpha-1} \psi(s) ;
$$

(iii) $\mathrm{G}(\mathrm{t}, \mathrm{s}) \geqslant 0$ for every $(\mathrm{t}, \mathrm{s}) \in[0,1] \times[0,1]$.

Proof. Properties (i) and (ii) follow immediately from the definition of the Green's function G. On the other hand, from (3.5), we have

$$
C(\lambda, \alpha, \beta, \gamma)>0
$$

Therefore, by (ii) we deduce (iii).

The following result is an immediate consequence of Lemma 3.4.

Lemma 3.6. Let $2<\alpha<3 \lambda>0$, and $\beta, \gamma \geqslant 0$ be such that (3.3) is satisfied. Let $y \in \mathrm{L}^{1}([\mathrm{a}, \mathrm{b}] ; \mathbb{R})$. Then the fractional boundary value problem

$$
\left\{\begin{array}{l}
D_{0}^{\alpha} u(t)+y(t)=0, \quad 0<t<1 \\
u(0)=u^{\prime}(0)=0, u(1)=\lambda\left(\left(I_{0}^{\beta} u\right)(1)+\left(I_{0}^{\gamma} u\right)(1)\right)
\end{array}\right.
$$

has a unique solution in $\mathrm{X}_{0}^{\alpha}([0,1] ; \mathbb{R})$, which is given by

$$
u(t)=\int_{0}^{1} G(t, s) y(s) d s, \quad t \in[0,1] .
$$


Now, we are able to give an equivalent integral formulation of problem (1.1). problem (1.1) is investigated under the following assumptions:

(A1) $2<\alpha<3$ and $\beta, \gamma \geqslant 0$;

(A2) (3.5) is satisfied;

(A3) $f:[0,1] \times[0, \infty) \times[0, \infty) \rightarrow[0, \infty)$ and $g:[0,1] \times[0, \infty) \rightarrow[0, \infty)$ are continuous functions;

(A4) $\mathrm{H}: \mathrm{C}([0,1] ; \mathbb{R}) \rightarrow \mathrm{C}([0,1] ; \mathbb{R})$ satisfies

$$
u \in \mathrm{C}([0,1] ;[0, \infty)) \Longrightarrow \mathrm{Hu} \in \mathrm{C}([0,1] ;[0, \infty)) .
$$

For $u \in C([0,1] ;[0, \infty))$, we denote by $\mathrm{Tu}$ the function defined by

$$
(T u)(t)=\int_{0}^{1} G(t, s)[f(s, u(s),(H u)(s))+g(s, u(s))] d s, \quad t \in[0,1] .
$$

From the above assumptions and the properties (i) and (iii) of Lemma 3.5, it can be easily seen that

$$
\mathrm{T}: \mathrm{C}([0,1] ;[0, \infty)) \rightarrow \mathrm{C}([0,1] ;[0, \infty))
$$

is a well-defined operator. Moreover, from Lemma 3.6, $u \in X_{0}^{\alpha}([0,1] ; \mathbb{R})$ is a solution of (1.1) if and only if $u \in C([0,1] ;[0, \infty))$ is a fixed point of $\mathrm{T}$.

\section{The mixed monotone operator method}

From the above study, we know that $u \in X_{0}^{\alpha}([0,1] ; \mathbb{R})$ is a solution of (1.1) if and only if $u \in$ $\mathrm{C}([0,1] ;[0, \infty))$ is a fixed point of the operator $\mathrm{T}$ defined by (3.6). In order to study the existence of fixed points of the operator $\mathrm{T}$, we shall use the mixed monotone operator method. In this section, we recall the basic tools related to this method.

Let $(E,\|\cdot\|)$ be a real Banach space. We denote by $\theta_{E}$ the zero vector of $E$.

Definition 4.1. A nonempty closed convex subset $P$ of $E$ is said to be a cone in $E$ if it satisfies the following conditions:

(i) $(r, x) \in[0, \infty) \times P \Longrightarrow r x \in P$;

(ii) $(x,-x) \in P \times P \Longrightarrow x=\theta_{E}$.

Let $\mathrm{P}$ be a cone in $\mathrm{E}$. Then $\mathrm{P}$ induces a partial order $\leqslant_{\mathrm{P}}$ in $\mathrm{E}$ defined by

$$
(x, y) \in E \times E, \quad x \leqslant P y \Longleftrightarrow y-x \in P .
$$

By $x<p y,(x, y) \in E \times E$, we mean $x \leqslant p \quad y$ and $x \neq y$.

Definition 4.2. Let $P$ be a cone in $E$. If there exists a constant $C>0$ such that

$$
(x, y) \in E \times E, \theta_{E} \leqslant p x \leqslant p \quad y\|x\| \leqslant C\|y\|,
$$

then the cone $\mathrm{P}$ is said to be normal. In this case, the smallest constant $\mathrm{C}$ satisfying the above property is called the normal constant of $P$.

For $(x, y) \in E \times E$, the notation $x \sim y$ means that there exist constants $\rho, \tau>0$ such that

$$
\tau y \leqslant_{p} x \leqslant_{p} \rho y .
$$

It can be easily seen that $\sim$ is an equivalence relation in $E$.

For $h \in E, \theta_{E}<_{P} h$, we denote by $P_{h}$ the subset of $P$ defined by

$$
P_{h}=\{x \in P: x \sim h\} .
$$

For more details on cones in Banach spaces, we refer the reader to [14]. 
Definition 4.3. An operator $S: E \rightarrow E$ is said to be increasing (Resp. decreasing) if for every $(x, y) \in E \times E$, $x \leqslant p$ y implies that $S x \leqslant p$ Sy (Resp. Sy $\left.\leqslant_{p} S x\right)$.

Definition 4.4. An operator $A: P \times P \rightarrow P$ is said to be mixed monotone if $A(x, y)$ is increasing in $x$, and decreasing in $y$, i.e.,

$$
(x, y),(u, v) \in P \times P, x \leqslant P u, y \geqslant_{P} v \Longrightarrow A(x, y) \leqslant_{P} A(u, v) .
$$

Definition 4.5. An operator $\mathrm{B}: \mathrm{P} \rightarrow \mathrm{P}$ is said to be subhomogeneous if

$$
(t, x) \in(0,1) \times P \Longrightarrow B(t x) \geqslant_{P} t B x .
$$

The following result appears in [29], and it is the main tool used in our study.

Lemma 4.6. Let $(\mathrm{E},\|\cdot\|)$ be a real Banach space, and let $\mathrm{P}$ be a normal cone in $\mathrm{E}$. Let $\mathrm{h} \in \mathrm{E}, \theta_{\mathrm{E}}<_{\mathrm{P}} \mathrm{h}$, and $\tau \in(0,1)$. Let us consider two operators $\mathrm{A}: \mathrm{P} \times \mathrm{P} \rightarrow \mathrm{P}$ and $\mathrm{B}: \mathrm{P} \rightarrow \mathrm{P}$. We suppose that $\mathrm{A}$ is a mixed monotone operator satisfying

$$
A\left(t x, t^{-1} y\right) \geqslant_{P} t^{\tau} A(x, y), \quad t \in(0,1),(x, y) \in P \times P .
$$

We suppose also that $\mathrm{B}$ is an increasing subhomogeneous operator satisfying the following conditions:

(i) there exists $h_{0} \in P$ such that $\mathrm{A}\left(\mathrm{h}_{0}, \mathrm{~h}_{0}\right) \in \mathrm{P}_{\mathrm{h}}$ and $\mathrm{Bh} \mathrm{h}_{0} \in \mathrm{P}_{\mathrm{h}}$;

(ii) there exists a constant $\delta_{0}>0$ such that

$$
A(x, y) \geqslant_{P} \delta_{0} B x, \quad(x, y) \in P \times P .
$$

Then

(a) $\mathrm{A}: \mathrm{P}_{\mathrm{h}} \times \mathrm{P}_{\mathrm{h}} \rightarrow \mathrm{P}_{\mathrm{h}}$ and $\mathrm{B}: \mathrm{P}_{\mathrm{h}} \rightarrow \mathrm{P}_{\mathrm{h}}$;

(b) there exist $\mathrm{u}_{0}, v_{0} \in \mathrm{P}_{\mathrm{h}}$ and $\mathrm{r} \in(0,1)$ such that

$$
r v_{0} \leqslant p u_{0}<_{p} v_{0}, u_{0} \leqslant p A\left(u_{0}, v_{0}\right)+B u_{0} \leqslant p A\left(v_{0}, u_{0}\right)+B v_{0} \leqslant p v_{0} ;
$$

(c) there exists a unique solution $x^{*} \in \mathrm{P}_{\mathrm{h}}$ such that

$$
x^{*}=A\left(x^{*}, x^{*}\right)+B x^{*} ;
$$

(d) for any initial values $\mathrm{x}_{0}, \mathrm{y}_{0} \in \mathrm{P}_{\mathrm{h}}$, constructing successively the sequences

$$
x_{n}=A\left(x_{n-1}, y_{n-1}\right)+B x_{n-1}, y_{n}=A\left(y_{n-1}, x_{n-1}\right)+B y_{n-1}, \quad n=1,2, \ldots,
$$

we have $\left\|x_{n}-x^{*}\right\| \rightarrow 0$ and $\left\|y_{n}-x^{*}\right\| \rightarrow 0$ as $\mathrm{n} \rightarrow \infty$.

Now, we are ready to prove our main result.

\section{Main result}

In this section, sufficient conditions are provided for the existence and uniqueness of positive solutions for problem (1.1). An iterative algorithm is also provided in order to approximate the solution.

We will work in the Banach space $C([0,1] ; \mathbb{R})$ equipped with the standard norm $\|\cdot\|_{\infty}$ defined by

$$
\|u\|_{\infty}=\max \{|u(t)|: 0 \leqslant t \leqslant 1\}, \quad u \in C([0,1] ; \mathbb{R}) .
$$

In $C([0,1] ; \mathbb{R})$, we consider the cone $\mathrm{P}=\mathrm{C}([0,1] ;[0, \infty))$. It can be easily seen that $\mathrm{P}$ is a normal cone with normal constant equal to 1 . The partial order $\leqslant \mathrm{P}$ induced by the cone $\mathrm{P}$ is defined by

$$
(\mathrm{u}, v) \in \mathrm{C}([0,1] ; \mathbb{R}) \times \mathrm{C}([0,1] ; \mathbb{R}), \quad \mathrm{u} \leqslant \mathrm{p} v \Longleftrightarrow \mathrm{u}(\mathrm{t}) \leqslant v(\mathrm{t}), 0 \leqslant \mathrm{t} \leqslant 1 .
$$

The following additional assumptions are needed. 
(A5) There exists $t_{0} \in[0,1]$ such that $g\left(t_{0}, 0\right)>0$.

(A6) For fixed $t \in[0,1], f(t, x, y)$ is increasing in $x$, and decreasing in $y$; and $g(t, x)$ is increasing in $x$.

(A7) For every $(k, t, x) \in(0,1) \times[0,1] \times[0, \infty)$, we have

$$
g(t, k x) \geqslant k g(t, x)
$$

(A8) There exists a constant $\tau \in(0,1)$ such that

$$
f\left(t, k x, k^{-1} y\right) \geqslant k^{\tau} f(t, x, y), \quad(k, t, x, y) \in(0,1) \times[0,1] \times[0, \infty) \times[0, \infty) .
$$

(A9) There exists a constant $\delta_{0}>0$ such that

$$
f(t, x, y) \geqslant \delta_{0} g(t, x), \quad(t, x, y) \in[0,1] \times[0, \infty) \times[0, \infty) .
$$

(A10) $\mathrm{H}: \mathrm{C}([0,1] ; \mathbb{R}) \rightarrow \mathrm{C}([0,1] ; \mathbb{R})$ satisfies

(H1) $(u, v) \in \mathrm{P} \times \mathrm{P}, \mathrm{u} \leqslant \mathrm{P} v \Longrightarrow \mathrm{Hu} \leqslant \mathrm{P} H v$;

$(H 2) H(k u) \geqslant_{p} k H u$ for every $(k, u) \in(0,1) \times P$.

Our main result in this paper is the following.

Theorem 5.1. Suppose that assumptions (A1)-(A10) are satisfied. Let $\mathrm{h} \in \mathrm{C}([0,1] ; \mathbb{R})$ be the function defined by $h(t)=t^{\alpha-1}, t \in[0,1]$. Then

(i) there exist $\mathrm{u}_{0}, v_{0} \in \mathrm{P}_{\mathrm{h}}$ and $\mathrm{r} \in(0,1)$ such that

$$
r v_{0} \leqslant \mathrm{p} u_{0}<_{p} v_{0}
$$

and, moreover, for every $\mathrm{t} \in[0,1]$, we have

$$
\begin{aligned}
& u_{0}(t) \leqslant \int_{0}^{1} G(t, s)\left[f\left(s, u_{0}(s),\left(H v_{0}\right)(s)\right)+g\left(s, u_{0}(s)\right)\right] d s, \\
& v_{0}(t) \geqslant \int_{0}^{1} G(t, s)\left[f\left(s, v_{0}(s),\left(H u_{0}\right)(s)\right)+g\left(s, v_{0}(s)\right)\right] d s ;
\end{aligned}
$$

(ii) problem (1.1) has a unique positive solution $\mathrm{x}^{*} \in \mathrm{P}_{\mathrm{h}}$;

(iii) for any initial values $x_{0}, y_{0} \in P_{h}$, constructing successively the sequences

$$
x_{n}(t)=\int_{0}^{1} G(t, s)\left[f\left(s, x_{n-1}(s),\left(H y_{n-1}\right)(s)\right)+g\left(s, x_{n-1}(s)\right)\right] d s, \quad t \in[0,1], n=1,2, \ldots
$$

and

$$
y_{n}(t)=\int_{0}^{1} G(t, s)\left[f\left(s, y_{n-1}(s),\left(H x_{n-1}\right)(s)\right)+g\left(s, y_{n-1}(s)\right)\right] d s, \quad t \in[0,1], n=1,2, \ldots,
$$

we have $\left\|\mathrm{x}_{\mathrm{n}}-\mathrm{x}^{*}\right\|_{\infty} \rightarrow 0$ and $\left\|\mathrm{y}_{\mathrm{n}}-\mathrm{x}^{*}\right\|_{\infty} \rightarrow 0$ as $\mathrm{n} \rightarrow \infty$.

Proof. It was shown in Section 3 that $u \in X_{0}^{\alpha}([0,1] ; \mathbb{R})$ is a solution of (1.1) if and only if $u \in P$ is a fixed point of $\mathrm{T}$, where $\mathrm{T}: \mathrm{P} \rightarrow \mathrm{P}$ is defined by (3.6). Let us introduce the operators $\mathrm{A}: \mathrm{P} \times \mathrm{P} \rightarrow \mathrm{P}$ and $\mathrm{B}: \mathrm{P} \rightarrow \mathrm{P}$ defined by

$$
A(u, v)(t)=\int_{0}^{1} G(t, s) f(s, u(s),(H v)(s)) d s, \quad(u, v) \in P \times P, t \in[0,1]
$$

and

$$
(B u)(t)=\int_{0}^{1} G(t, s) g(s, u(s)) d s, \quad u \in P, t \in[0,1]
$$


Observe that $u \in P$ is a fixed point of $T$ if and only if $u \in P$ satisfies the operator equation $A(u, u)+B u=$ u.

In the sequel, we check that assumptions of Lemma 4.6 are satisfied. From (A6), (H1), and the positivity of the Green's function $G$ (see property (iii) in Lemma 3.5), it is easily seen that $A$ is a mixed monotone operator and $\mathrm{B}$ is an increasing operator. Let $k \in(0,1)$ and $(x, y) \in \mathrm{P} \times \mathrm{P}$. We have

$$
A\left(k x, k^{-1} y\right)(t)=\int_{0}^{1} G(t, s) f\left(s, k x(s),\left(H\left(k^{-1} y\right)\right)(s)\right) d s, \quad t \in[0,1] .
$$

On the other hand, from $(\mathrm{H} 2)$ we have

$$
H y=H\left(k\left(k^{-1} y\right)\right) \geqslant_{P} k H\left(k^{-1} y\right),
$$

that is,

$$
H\left(k^{-1} y\right)(s) \leqslant k^{-1}(H y)(s), \quad s \in[0,1] .
$$

Using the above inequality, (A6), and the positivity of the Green's function G, we obtain

$$
A\left(k x, k^{-1} y\right)(t) \geqslant \int_{0}^{1} G(t, s) f\left(s, k x(s), k^{-1}(H y)(s)\right) d s, \quad t \in[0,1],
$$

which implies by (A8) that

$$
A\left(k x, k^{-1} y\right)(t) \geqslant k^{\tau} \int_{0}^{1} G(t, s) f(s, x(s),(H y)(s)) d s=k^{\tau} A(x, y)(t), \quad t \in[0,1] .
$$

Therefore, condition (4.1) of Lemma 4.6 is satisfied. Next, by assumption (A7) and the positivity of the Green's function $G$, we see easily that B is a subhomogeneous operator .

Next, we take the function

$$
h(t)=t^{\alpha-1}, \quad t \in[0,1] .
$$

Clearly, we have $h \in P$ and $h \not \equiv 0$. Taking into account property (ii) in Lemma 3.5, assumptions (A4) and (A6), and the positivity of the Green's function $G$, we get

$$
A(h, h)(t)=\int_{0}^{1} G(t, s) f(s, h(s),(H h)(s)) d s \leqslant \int_{0}^{1} G(t, s) f(s, 1,0) d s \leqslant t^{\alpha-1} \int_{0}^{1} v(s) f(s, 1,0) d s, \quad t \in[0,1],
$$

that is,

$$
A(h, h) \leqslant p\left(\int_{0}^{1} v(s) f(s, 1,0) d s\right) h .
$$

Similarly, using the above assumptions and (H1), we obtain

$$
\begin{aligned}
A(h, h)(t) & =\int_{0}^{1} G(t, s) f(s, h(s),(H h)(s)) d s \\
& \geqslant \int_{0}^{1} G(t, s) f(s, 0,(H 1)(s)) d s \geqslant t^{\alpha-1} \int_{0}^{1} \mu(s) f(s, 0,(H 1)(s)) d s, \quad t \in[0,1]
\end{aligned}
$$

that is,

$$
A(h, h) \geqslant_{P}\left(\int_{0}^{1} \mu(s) f(s, 0,(H 1)(s)) d s\right) h .
$$

Combining (5.1) with (5.2), we obtain

$$
\alpha_{1} h \leqslant_{P} A(h, h) \leqslant_{P} \alpha_{2} h,
$$


where

$$
\alpha_{1}=\int_{0}^{1} \mu(s) f(s, 0,(H 1)(s)) d s \quad \text { and } \quad \alpha_{2}=\int_{0}^{1} v(s) f(s, 1,0) d s .
$$

In what follows, we shall prove that $\alpha_{i}>0, i=1,2$. To do this, it is sufficient to prove that $\alpha_{1}>0$ (since $\left.\alpha_{1} \leqslant \alpha_{2}\right)$. In fact, since $g\left(t_{0}, 0\right)>0$ for some $t_{0} \in[0,1]$ (from assumption (A5)), the continuity of $g$ implies the existence of a certain subest $E$ of $[0,1]$ with a non-zero Lebesgue measure such that

$$
g(t, 0)>0, \quad t \in E .
$$

Now, taking in consideration assumption (A9), we have

$$
f(s, 0,(H 1)(s)) \geqslant \delta_{0} g(s, 0), \quad s \in[0,1] .
$$

Therefore, by (5.4) and (5.5), we have

$$
\begin{aligned}
\alpha_{1} & =\int_{0}^{1} \mu(s) f(s, 0,(\mathrm{H} 1)(s)) d s \\
& =\int_{0}^{1} \frac{C(\lambda, \alpha, \beta, \gamma)}{\Gamma(\alpha)}(1-s)^{\alpha-1}\left[\frac{\lambda}{\Gamma(\alpha+\beta)}\left(1-(1-s)^{\beta}\right)+\frac{\lambda}{\Gamma(\alpha+\gamma)}\left(1-(1-s)^{\gamma}\right)\right] \times f(s, 0,(H 1)(s)) d s \\
& \geqslant \frac{\delta_{0} C(\lambda, \alpha, \beta, \gamma)}{\Gamma(\alpha)} \int_{0}^{1}(1-s)^{\alpha-1}\left[\frac{\lambda}{\Gamma(\alpha+\beta)}\left(1-(1-s)^{\beta}\right)+\frac{\lambda}{\Gamma(\alpha+\gamma)}\left(1-(1-s)^{\gamma}\right)\right] \times g(s, 0) d s \\
& \geqslant \frac{\delta_{0} C(\lambda, \alpha, \beta, \gamma)}{\Gamma(\alpha)} \int_{E}(1-s)^{\alpha-1}\left[\frac{\lambda}{\Gamma(\alpha+\beta)}\left(1-(1-s)^{\beta}\right)+\frac{\lambda}{\Gamma(\alpha+\gamma)}\left(1-(1-s)^{\gamma}\right)\right] \times g(s, 0) d s>0 .
\end{aligned}
$$

Hence, we deduce by (5.3) that $A(h, h) \in P_{h}$.

Using similar arguments as above, for any $t \in[0,1]$, we have

$$
\beta_{1} h \leqslant_{P} B h \leqslant p \beta_{2} h,
$$

where

$$
\beta_{1}=\int_{0}^{1} \mu(s) g(s, 0) d s>0 \text { and } \beta_{2}=\int_{0}^{1} v(s) g(s, 1) d s .
$$

Therefore, we have $B h \in P_{h}$. Hence, assumption (i) of Lemma 4.6 is satisfied with $h_{0}=h$.

Now, let $(x, y) \in P \times P$. Using assumption (A9), we obtain

$$
A(x, y)(t)=\int_{0}^{1} G(t, s) f(s, x(s),(H y)(s)) d s \geqslant \delta_{0} \int_{0}^{1} G(t, s) g(s, x(s)) d s=\delta_{0}(B x)(t), \quad t \in[0,1] .
$$

Hence, we have

$$
A(x, y) \geqslant_{P} \delta_{0} B x, \quad(x, y) \in P \times P,
$$

and assumption (ii) of Lemma 4.6 is satisfied.

Finally, applying Lemma 4.6, the desired result follows.

\section{Examples}

In order to illustrate the obtained result given by Theorem 5.1, some examples are presented in this section.

Example 6.1. Consider the fractional boundary value problem

$$
\left\{\begin{array}{l}
D_{0}^{\frac{5}{2}} u(t)+t+t^{3}+2 \sqrt{u(t)}+\frac{1}{[u(t)]^{p}+1}=0, \quad 0<t<1 \\
u(0)=u^{\prime}(0)=0, u(1)=\int_{0}^{1} u(s) d s
\end{array}\right.
$$

where $0<p<1$. 
Problem (6.1) is a particular case of problem (1.1) with $\alpha=\frac{5}{2}, \lambda=\frac{1}{2}, \beta=\gamma=1, f(t, x, y)=t+\sqrt{x}+$ $\frac{1}{y^{p}+1}, g(t, x)=t^{3}+\sqrt{x}$, and $H: C([0,1] ; \mathbb{R}) \rightarrow C([0,1] ; \mathbb{R})$ is the operator defined by $\mathrm{Hu}=u$ for every $u \in C([0,1] ; \mathbb{R})$.

In this considered case, we have

$$
\lambda\left(\frac{1}{\Gamma(\alpha+\beta)}+\frac{1}{\Gamma(\alpha+\gamma)}\right)=\frac{1}{\Gamma\left(\frac{7}{2}\right)} \approx 0.3095 \quad \text { and } \quad \frac{1}{\Gamma(\alpha)}=\frac{1}{\Gamma\left(\frac{5}{2}\right)} \approx 0.7524
$$

Observe that

$$
0<\lambda\left(\frac{1}{\Gamma(\alpha+\beta)}+\frac{1}{\Gamma(\alpha+\gamma)}\right)<\frac{1}{\Gamma(\alpha)} .
$$

Therefore, assumptions (A1) and (A2) of Theorem 5.1 are satisfied. Moreover, it is clear that $f:[0,1] \times$ $[0, \infty) \times[0, \infty) \rightarrow[0, \infty)$ and $g:[0,1] \times[0, \infty) \rightarrow[0, \infty)$ are continuous functions, and then assumption (A3) is satisfied. Assumptions concerning the operator $\mathrm{H}((\mathrm{A} 4)$ and (A10)) are trivial. On the other hand, we have $g(1,0)=1>0$. Therefore, assumption (A5) is satisfied. It is easily seen that assumption (A6) is satisfied. In order to check assumption (A7), let us take $(k, t, x) \in(0,1) \times[0,1] \times[0, \infty)$. We have

$$
g(t, k x)=t^{3}+\sqrt{k x} \geqslant k t^{3}+\sqrt{k} \sqrt{x} \geqslant k t^{3}+k \sqrt{x}=k\left(t^{3}+\sqrt{x}\right)=k g(t, x) .
$$

Then assumption (A7) is satisfied. Next, let $(k, t, x, y) \in(0,1) \times[0,1] \times[0, \infty) \times[0, \infty)$. We have

$$
\begin{aligned}
f\left(t, k x, k^{-1} y\right)=t+\sqrt{k x}+\frac{1}{k^{-p} y^{p}+1} & =t+\sqrt{k} \sqrt{x}+\frac{k^{p}}{y^{p}+k^{p}} \\
& \geqslant t+\sqrt{k} \sqrt{x}+\frac{k^{p}}{y^{p}+1} \\
& \geqslant k^{\max \left\{\frac{1}{2}, p\right\}}\left(t+\sqrt{x}+\frac{1}{y^{p}+1}\right)=k^{\max \left\{\frac{1}{2}, p\right\}} f(t, x, y) .
\end{aligned}
$$

Hence assumption (A8) is satisfied with $\tau=\max \left\{\frac{1}{2}, p\right\}$. Finally, it can be easily seen that assumption (A9) is satisfied with $\delta_{0}=1$. Therefore, all assumptions of Theorem 5.1 are satisfied. As consequence, problem 6.1 admits a unique solution $x^{*} \in P_{h}$, where $h(t)=t^{\frac{3}{2}}, t \in[0,1]$.

Next, we present some examples of operators $\mathrm{H}: \mathrm{C}([0,1] ; \mathbb{R}) \rightarrow \mathrm{C}([0,1] ; \mathbb{R})$ satisfying assumptions (A4) and (A10) of Theorem 5.1.

Example 6.2. Let $\varphi:[0,1] \rightarrow[0,1]$ be a continuous function. Define the operator $\mathrm{H}: \mathrm{C}([0,1] ; \mathbb{R}) \rightarrow$ $\mathrm{C}([0,1] ; \mathbb{R})$ by

$$
(\mathrm{Hu})(\mathrm{t})=\mathrm{u}(\varphi(\mathrm{t})), \quad \mathrm{t} \in[0,1]
$$

for every $u \in C([0,1] ; \mathbb{R})$. Clearly, such operator satisfies assumptions (A4) and (A10).

Example 6.3. Let $\varphi:[0,1] \rightarrow[0, \infty)$ be a continuous function. Define the operator $\mathrm{H}: \mathrm{C}([0,1] ; \mathbb{R}) \rightarrow$ $\mathrm{C}([0,1] ; \mathbb{R})$ by

$$
(\mathrm{Hu})(\mathrm{t})=\varphi(\mathrm{t}) \mathrm{u}(\mathrm{t}), \quad \mathrm{t} \in[0,1]
$$

for every $u \in C([0,1] ; \mathbb{R})$. Then the operator $\mathrm{H}$ satisfies assumptions (A4) and (A10).

Example 6.4. Let $\varphi:[0,1] \rightarrow[0, \infty)$ be a continuous function. Define the operator $H: C([0,1] ; \mathbb{R}) \rightarrow$ $\mathrm{C}([0,1] ; \mathbb{R})$ by

$$
(H u)(t)=\varphi(t) u(t), \quad t \in[0,1]
$$

for every $u \in C([0,1] ; \mathbb{R})$. Then the operator $\mathrm{H}$ satisfies assumptions (A4) and (A10).

Notice that the composition and multiplication operators on $C([0,1] ; \mathbb{R})$ are linear. The next examples present nonlinear operators $\mathrm{H}: \mathrm{C}([0,1] ; \mathbb{R}) \rightarrow \mathrm{C}([0,1] ; \mathbb{R})$ satisfying the required assumptions. 
Example 6.5. For $u \in C([0,1] ; \mathbb{R})$, we define the mapping $\mathrm{Hu}:[0,1] \rightarrow \mathbb{R}$ by

$$
(\mathrm{Hu})(\mathrm{t})=\max \{|\mathrm{u}(\mathrm{s})|: 0 \leqslant s \leqslant \mathrm{t}\}, \quad \mathrm{t} \in[0,1] .
$$

It was proved in $[9]$ that $\mathrm{H}$ maps $\mathrm{C}([0,1] ; \mathbb{R})$ into itself. Moreover, it can be easily seen that assumptions (A4) and (A10) are satisfied by the operator $\mathrm{H}$.

Example 6.6. Let $r \in(0,1)$. For $u \in C([0,1] ; \mathbb{R})$, we define the mapping $H u:[0,1] \rightarrow \mathbb{R}$ by

$$
(H u)(t)=u^{r}(t), \quad t \in[0,1]
$$

It can be easily seen that assumptions (A4) and (A10) are satisfied by the operator $\mathrm{H}$.

Example 6.7. Using the same arguments as in Example 6.1, if $\mathrm{H}: \mathrm{C}([0,1] ; \mathbb{R}) \rightarrow \mathrm{C}([0,1] ; \mathbb{R})$ is any operator between the ones appearing in Examples 6.2-6.6, then the fractional boundary value problem

$$
\left\{\begin{array}{l}
D_{0}^{\frac{5}{2}} u(t)+t+t^{3}+2 \sqrt{u(t)}+\frac{1}{[(H u)(t)]^{p}+1}=0, \quad 0<t<1 \\
u(0)=u^{\prime}(0)=0, u(1)=\int_{0}^{1} u(s) d s
\end{array}\right.
$$

where $0<p<1$, admits a unique solution $x^{*} \in P_{h}$, where $h(t)=t^{\frac{3}{2}}, t \in[0,1]$.

\section{Comparison with existing results}

In [7], the authors considered the fractional boundary value problem

$$
\left\{\begin{array}{l}
D_{0}^{\alpha} \mathfrak{u}(t)+F(t, u(t))=0, \quad 0<t<1 \\
\mathfrak{u}(0)=u^{\prime}(0)=0, \mathfrak{u}(1)=\mu \int_{0}^{1} \mathfrak{u}(s) d s
\end{array}\right.
$$

where $2<\alpha<3,0<\mu<\alpha$ and $\mathrm{F}:[0,1] \times[0, \infty) \rightarrow[0, \infty)$. Observe that problem (6.1) is a particular case of problen (7.1) with $\alpha=\frac{5}{2}, \mu=1$ and $F(t, x)=t+t^{3}+2 \sqrt{x}+\frac{1}{x^{p}+1}$.

Sufficient conditions for the existence of solutions for problem (7.1) were provided in [7]. Before recalling the main result in [7], we need to introduce some notations.

We put

$$
F_{0}:=\lim _{x \rightarrow 0^{+}}\left(\min _{t \in\left[\frac{1}{2}, 1\right]} \frac{F(t, x)}{x}\right), F_{\infty}:=\lim _{x \rightarrow \infty}\left(\min _{t \in\left[\frac{1}{2}, 1\right]} \frac{F(t, x)}{x}\right)
$$

and

$$
F^{0}:=\lim _{x \rightarrow 0^{+}}\left(\max _{t \in[0,1]} \frac{F(t, x)}{x}\right), F^{\infty}:=\lim _{x \rightarrow \infty}\left(\max _{t \in[0,1]} \frac{F(t, x)}{x}\right)
$$

Theorem 7.1 ([7]). Suppose that

(a) $\mathrm{F}:[0,1] \times[0, \infty) \rightarrow[0, \infty)$ is continuous;

(b) $\left(\mathrm{F}_{0}, \mathrm{~F}^{\infty}\right)=(\infty, 0)$ (sublinear case) or $\left(\mathrm{F}^{0}, \mathrm{~F}_{\infty}\right)=(0, \infty)$ (superlinear case).

Then problem (7.1) admits at least one positive solution in $\mathrm{C}([0,1] ; \mathbb{R})$.

Notice that in Example 6.1, we have

$$
\min _{\mathbf{t} \in\left[\frac{1}{2}, 1\right]} \frac{F(t, x)}{x}=\frac{\frac{1}{2}+\frac{1}{8}+2 \sqrt{x}+\frac{1}{x^{p}+1}}{x} \text { and } \max _{\mathbf{t} \in[0,1]} \frac{F(t, x)}{x}=\frac{2+2 \sqrt{x}+\frac{1}{x^{p}+1}}{x} .
$$


In such a case, it can be easily seen that

$$
\left(F_{0}, F^{\infty}\right)=(\infty, 0) \text { and }\left(F^{0}, F_{\infty}\right)=(\infty, 0) .
$$

Therefore, we are in the sublinear case. Hence, by Theorem 7.1, problem (6.1) admits at least one positive solution in $\mathrm{C}([0,1] ; \mathbb{R})$.

Observe that Theorem 7.1 provides only the existence of positive solutions for problem (6.1), while by our result (Theroem 5.1), we have an additional information, that is, a unique solution of problem 6.1 exists in the set $P_{h}$, where $h(t)=t^{\frac{3}{2}}, t \in[0,1]$.

\section{Acknowledgment}

I. Azman, M. Jleli and B. Samet extend their appreciation to the Deanship of Scientific Research at King Saud University for funding this work through research group No RGP-034. K. Sadarangani is supported by the project MTM-2016-49736-P.

\section{References}

[1] R. P. Agarwal, B. Ahmad, Existence theory for anti-periodic boundary value problems of fractional differential equations and inclusions, Comput. Math. Appl., 62 (2011), 1200-1214. 1

[2] B. Ahmad, A. Alsaedi, B. Alghamdi, Analytic approximation of solutions of the forced Duffing equation with integral boundary conditions, Nonlinear Anal. Real World Appl., 9 (2008), 1727-1740. 1

[3] B. Ahmad, J. J. Nieto, Existence results for nonlinear boundary value problems of fractional integrodifferential equations with integral boundary conditions, Bound. Value Probl., 2009 (2009), 11 pages.

[4] B. Ahmad, S. K. Ntouyas, Existence results for higher order fractional differential inclusions with multi-strip fractional integral boundary conditions, Electron. J. Qual. Theory Differ. Equ., 9 (2013), 51-60.

[5] B. Ahmad, S. Sivasundaram, Existence of solutions for impulsive integral boundary value problems of fractional order, Nonlinear Anal. Hybrid Syst., 4 (2010), 134-141. 1

[6] E. Barkai, R. Metzler, J. Klafter, From continuous time random walks to the fractional Fokker-Planck equation, Phys. Rev. E., 61 (2000), 132-138. 1

[7] A. Cabada, Z. Hamdi, Nonlinear fractional differential equations with integral boundary value conditions, Appl. Math. Comput., 228 (2014), 251-257. 1, 7, 7, 7.1

[8] A. Cabada, G. Wang, Positive solutions of nonlinear fractional differential equations with integral boundary value conditions, J. Math. Anal. Appl., 389 (2012), 403-411. 1

[9] J. Caballero, B. López, K. Sadarangani, On monotonic solutions of an integral equation of Volterra type with supremum, J. Math. Anal. Appl., 305 (2005), 304-315. 6.5

[10] G. Chai, Existence results for boundary value problems of nonlinear fractional differential equations, Comput. Math. Appl., 62 (2011), 2374-2382. 1

[11] Y. S. Choi, K.-Y. Chan, A parabolic equation with nonlocal boundary conditions arising from electrochemistry, Nonlinear Anal., 18 (1992), 317-331. 1

[12] L. Debnath, Recent applications of fractional calculus to science and engineering, Int. J. Math. Math. Sci., 54 (2003), 3413-3442. 1

[13] N. M. Fonseca Ferreira, F. B. Duarte, M. F. M. Lima, M. G. Marcos, J. A. Machado Tenreiro, Application of fractional calculus in the dynamical analysis and control of mechanical manipulators, Fract. Calc. Appl. Anal., 11 (2008), 91-113. 1

[14] D. J. Guo, V. Lakshmikantham, Nonlinear Problems in Abstract Cones, Academic Press, Boston, (1988). 4

[15] R. Hilfer, Applications of Fractional Calculus in Physics, World Scientific, Singapore, (2000). 1

[16] D. Idczak, M. Majewski, Fractional fundamental lemma of order $\alpha \in\left(\mathrm{n}-\frac{1}{2}, \mathrm{n}\right)$ with $\mathrm{n} \in \mathbb{N}, \mathrm{n} \geqslant 2$, Dynam. Systems Appl., 21 (2012), 251-268. 2.3, 2.5, 2.6

[17] M. Jia, X. Liu, Three nonnegative solutions for fractional differential equations with integral boundary conditions, Comput. Math. Appl., 62 (2011), 1405-1412. 1

[18] M. Jleli, B. Samet, Existence of positive solutions to an arbitrary order fractional differential equation via a mixed monotone operator method, Nonlinear Anal. Model. Control, 20 (2015), 367-376. 1

[19] A. A. Kilbas, H. M. Srivastava, J. J. Trujillo, Theory and Applications of Fractional Differential Equations, Elsevier Science, Amsterdam, (2006). 1, 2.1, 2.2, 2.4

[20] X. Liu, M. Jia, Multiple solutions for fractional differential equations with nonlinear boundary conditions, Comput. Math. Appl., 59 (2010), 2880-2886. 1

[21] R. Magin, Fractional calculus in Bioengineering, Crit. Rev. Biomed. Eng., 32 (2004), 1-104. 1

[22] I. Podlubny, Fractional Differential Equations Mathematics in Science and Engineering, Academic Press, New York, (1999). 1 
[23] I. Podlubny, Geometric and physical interpretation of fractional integration and fractional differentiation, Fract. Calc. Appl. Anal., 5 (2002), 367-386. 1

[24] P. Shi, Weak solution to evolution problem with a nonlocal constraint, SIAM J. Math. Anal., 24 (1993), 46-58. 1

[25] P. Shi, M. Shillor, Design of contact patterns in one-dimensional thermoelasticity, Theoretical Aspects of Industrial Design, Philadelphia, (1992). 1

[26] W. Sudsutad, J. Tariboon, Existence results of fractional integrodifferential equations with m-point multi-term fractional order integral boundary conditions, Bound. Value Probl., 2012 (2012), 11 pages. 1

[27] G. Wang, W. Liu, C. Ren, Existence of solutions for multi-point nonlinear differential equations of fractional orders with integral boundary conditions, Electron. J. Differential Equations, 2012 (2012), 10 pages.

[28] R. Yan, S. Sun, H. Lu, Y. Zhao, Existence of solutions for fractional differential equations with integral boundary conditions, Adv. Difference Equ., 2014 (2014), 13 pages.

[29] C. Zhai, M. Hao, Fixed point theorems for mixed monotone operators with perturbation and applications to fractional differential equation boundary value problems, Nonlinear Anal., 75 (2012), 2542-2551. 1, 1, 4

[30] Y. Zhao, S. Sun, Z. Han, Q. Li, The existence of multiple positive solutions for boundary value problems of nonlinear fractional differential equations, Commun. Nonlinear Sci. Numer. Simul., 16 (2011), 2086-2097. 1 